Proyecciones Journal of Mathematics

Vol. 37, No 3, pp. 429-438, September 2018.

Universidad Católica del Norte

Antofagasta - Chile

\title{
Properly efficient solutions to non-differentiable multiobjective optimization problems
}

\author{
L. Batista dos Santos* \\ Universidade Federal do Paraná, Brasil \\ M. A. Rojas-Medar ${ }^{\dagger}$ \\ Universidad de Tarapacá, Chile \\ and \\ V. Vivanco-Orellana \\ Universidad Católica de la Santísima Concepción, Chile \\ Received: July 2017. Accepted : August 2018
}

\begin{abstract}
In this work sufficient conditions are established to ensure that all feasible points are (properly) efficient solutions in non trivial situations, for a class of non-differentiable, non-convex multiobjective minimization problems. Considering locally Lipschitz functions and some results of non-differentiable analysis introduced by F. H. Clarke [2].
\end{abstract}

Mathematics Subject Classification 2000 : 90C29, 90C26.

Keywords : Multiobjective optimization, efficient solution, properly efficient solution.

${ }^{*}$ This work is partially supported by CNPq $472313 / 2011-8$ and M.E.C. (Spain), Project MTM2010-15383.

${ }^{\dagger}$ This work was partially supported by Ministerio de Economía y Competitividad (Spain) Grant MTM2015-66185-P. 


\section{Introduction}

Daily we come across situations that lead to making decisions. Most of often have two or more objectives to be met simultaneously and that possibly are in conflict with each other. In these cases, often several objectives are fixed as goals by the decision maker. Typically, this type of problem arises in areas such as Business Management, Administration Resources, Planning Strategies, etc. The applicability of multiobjective problems is very extended and hence our interest in studying it.

Formally, the multiobjective optimization problem admits the following formulation:

$$
\left.\begin{array}{l}
\min f(x)=\left(f_{1}(x), \cdots, f_{n}(x)\right) \\
\text { s.t. : } x \in X,
\end{array}\right\}(\mathbf{P})
$$

where $f_{j}: X \rightarrow \mathbf{R}$ and $X$ is a non-empty subset of $\mathbf{R}^{n}$.

The first results in the field of multiobjective optimization are attributed to V. Pareto [5] and are related to the theory of Social Welfare. V. Pareto introduced the concept of efficient solution to the problem $(\mathbf{P})$. Informally, we say that a point $x^{*}$ in $X$ is an efficient solution of $(\mathbf{P})$ if it is not possible to improve any objective $f_{j}$ without worsening some other objective. Various modifications of this concept have arisen since that time. Another concept of solution is the properly efficiency, introduced by A. Geoffrion in [3]. Informally, a solution is properly efficient when the quotients, between gain and loss of an object relative to other, are bounded.

Denote by $P(X)$ the set of properly efficient solutions of $(\mathbf{P})$. We are interested in studying the cases when all points of the domain $X$ are (properly) efficient solutions. It is evident that this will take place if we restrict the domain $X$ to $Y \subseteq X$, such that $Y=P(X)$. This is the trivial case in which we obtain $Y=P(Y)$. We study some non-trivial situations where it is achieved $P(X)=X$.

Siposová [7] establishes conditions to ensure that all points of domain $X$ are properly efficient solutions, for continuously differentiable convex problems in nontrivial situations.

We extended the results obtained by Siposová in [7] for a class of nondifferentiable non-convex problems, considering locally Lipschitz functions and some results of non-differentiable analysis introduced by F. H. Clarke [2]. We consider the case where the functions defining the problem $(\mathbf{P})$ are invex and non-differentiable, such functions were introduced by Phuong, Sach and Yen in [6]. 


\section{Preliminaries}

The following convention for equalities and inequalities will be adopted. If $x=\left(x_{1}, \cdots, x_{m}\right), y=\left(y_{1}, \cdots, y_{m}\right)$ in $\mathbf{R}^{m}$ then

$$
\begin{aligned}
& x<y \Leftrightarrow x_{i}<y_{i}, \\
& x y \Leftrightarrow x_{i} \leq y_{i}, \quad \forall i=1, \cdots, m, \\
& x \leq y \Leftrightarrow x y, \exists r, x_{r}<y_{r} .
\end{aligned}
$$

The solution of the problem depends on the notion of "equilibrium" used to resolve conflicts that arise from simultaneous consideration of several objectives. The notions that we will adopt in this work are the notions of efficient and properly efficient solution [3].

Definition 1. A feasible point $x^{*}$ in $X$ is said to be efficient solution of (P) iff there not $x$ in $X$ such that $f(x) \leq f\left(x^{*}\right)$.

Definition 2. A feasible point $x^{*}$ in $X$ is said to be properly efficient solution (or Geoffrion-properly Efficient) of $(\mathbf{P})$ if it is efficient and there exists a constant $d>0$ such that, for every $i \in J$

$$
\frac{f_{i}(x)-f_{i}\left(x^{*}\right)}{f_{j}\left(x^{*}\right)-f_{j}(x)} \leq d
$$

for some $j \in J, j \neq r$ such that $f_{j}\left(x^{*}\right)<f_{j}(x)$ whenever $x \in X$ and $f_{i}\left(x^{*}\right)>f_{i}(x)$.

This last definition is equivalent to: $x^{*} \in X$ is properly efficient solution of (P) if there exists a constant $d>0$ such that, for each $x \in X, x \neq x^{*}$ and $i \in J$ there exists $j \in J, f_{j}\left(x^{*}\right)<f_{j}(x)$ such that

$$
f_{i}(x)+d f_{j}(x) \geq f_{i}\left(x^{*}\right)+d f_{j}\left(x^{*}\right) .
$$

\subsection{Non-differentiable Analysis}

Our notation is standard: Euclidean n-space $\mathbf{R}^{n}$ is provided by the usual inner product which we denote by $\langle\cdot, \cdot\rangle$ and the norm associated with this product will be denoted $\|\cdot\|$. Also we assume that this space is provided with the topology induced by norm $\|\cdot\|$. Let us recall some notions of non-differentiable analysis from [2]. 
Let $\Omega$ be a non-empty open subset of $\mathbf{R}^{n}$. The function $\phi: \Omega \rightarrow \mathbf{R}$ is said to be Lipschitz next to $x$ in $\Omega$ if there exists $\delta>0$ and $k=k(x, \delta)>0$ such that

$$
\left|\phi\left(x_{1}\right)-\phi\left(x_{2}\right)\right| \leq k|| x_{1}-x_{2} \|, \text { for all } x_{1}, x_{2} \in \Omega \cap B(x, \delta),
$$

where $B(x, \delta)$ is the open ball of center $x$ and ratio $\delta$.

If $\phi$ is Lipschitz next to each $x \in \Omega$, we say that $\phi$ is locally Lipschitz in $\Omega$.

Suppose that the function $\phi: \Omega \rightarrow \mathbf{R}$ is locally Lipschitz on $\Omega$. The generalized directional derivative of $\phi$ at $x$ in the direction $v \in \mathbf{R}^{n}$ denoted $\phi^{\circ}(x ; v)$ is defined

$$
\phi^{\circ}(x ; v):=\limsup _{\substack{y \rightarrow x \\ \lambda \downarrow 0}} \frac{\phi(y+\lambda v)-\phi(y)}{\lambda},
$$

and the generalized gradient of $\phi$ in $x$ is

$$
\partial \phi(x)=\left\{\xi \in \mathbf{R}^{n}: \phi^{0}(x ; v) \geq\langle\xi, v\rangle, \forall v \in \mathbf{R}^{n}\right\} .
$$

The following proposition establishes some nice properties of the directional derived and generalized gradient.

Proposition 3. Let $\phi: \Omega \rightarrow \mathbf{R}$ be a Lipschitz locally function on $\Omega$ with constant $k$. Then:

1. The function $v \mapsto \phi^{0}(x ; v)$ is finite, sublinear and satisfies $\left|\phi^{0}(x ; v)\right| \leq$ $k\|v\|$.

2. The subdifferential $\partial \phi(x)$ is nonempty compact convex set for every $x \in \Omega$, and $\|\xi\| \leq k, \quad \forall \xi \in \partial \phi(x)$.

3. For each $v \in \mathbf{R}^{n}, \phi^{0}(x ; v)=\max \{\langle\xi, v\rangle: \xi \in \partial \phi(x)\}$.

4. $\xi \in \partial \phi(x)$ if and only if $\phi^{0}(x ; v) \geq\langle\xi, v\rangle, \forall v \in \mathbf{R}^{n}$.

To obtain optimality conditions in terms of these concepts, we will need the notions of tangent and normal vectors.

Initially, we observe that if $C$ is a nonempty subset of $\mathbf{R}^{n}$, then the distance function $d_{C}: \mathbf{R}^{n} \rightarrow \mathbf{R}$, defined by $d_{C}(x)=\inf _{y \in C}\|y-x\|$ is globally Lipschitz, with Lipschitz constant equal to 1 , but it is not differentiable. 
In terms of the directional derivative of distance function it is possible to define the geometric notions tangent vector, without convexity assumption.

Let $x \in C$ and $v \in \mathbf{R}^{n}$ be. We say that $v$ is a tangent vector to $C$ at $x$ if $d^{0}(x ; v)=0$. The set of tangent vectors, denoted $T_{C}(x)$, is called tangent cone to $C$ at $x$ (of Clarke).

By polarity is defined the normal cone (of Clarke),

$$
N_{C}(x)=\left\{\xi \in \mathbf{R}^{n}:\langle\xi, v\rangle \leq 0, \forall v \in T_{C}(x)\right\}
$$

When $C$ is a convex set, these cones coincide with usual the tangent and normal cone, of Convex Analysis. In addition, for every $x \in C$ these cones are closed and convex.

Finally, we recall a result that establishes necessary optimality conditions in terms of a stationary condition.

Proposition 4. Let $\phi: \Omega \subseteq \mathbf{R}^{n} \rightarrow \mathbf{R}$ be a function defined on the open set $\Omega$ and let $C$ be a nonempty subset of $\Omega$. If $x^{*} \in C$ is a minimum of $\phi$, then

$$
0 \in \partial \phi\left(x^{*}\right)+N_{C}\left(x^{*}\right)
$$

A point $x^{*} \in C$ that satisfies $(2.2)$ is called stationary point of $\phi$ in $C$.

Remark 5. Let $C$ be a non-empty subset of $\Omega$.

i) If $x^{*} \in C$ is stationary point of $\phi$ then $\phi^{0}\left(x^{*} ; v\right) \geq 0, \forall v \in T_{C}\left(x^{*}\right)$.

ii) If $C=\Omega=\mathbf{R}^{n}$ then $T_{C}\left(x^{*}\right)=\mathbf{R}^{n}$ and $N_{C}\left(x^{*}\right)=\{0\}$ and if $x^{*} \in C$ is stationary point of $\phi$ then $0 \in \partial \phi\left(x^{*}\right)$.

iii) If $\phi$ is a continuously differentiable function in $C$, where $C$ is an open subset $\mathbf{R}^{n}$, then $\partial \phi\left(x^{*}\right)=\left\{\nabla \phi\left(x^{*}\right)\right\}$ and, if $x^{*} \in C$ is a stationary point of $\phi$ then $\nabla \phi\left(x^{*}\right)=0$.

\subsection{Generalized Convexity}

The optimality necessary conditions established by Proposition 4 are not sufficient for optimality, without additional assumptions about the functions that define the problem. If the functions involved in the problem are convex, which determines a convex problem, the necessary optimality conditions are also sufficient, but this assumption is very restrictive, since a wide class of problems are not convex. 
In order to weaken the convexity assumption, emerged in the related literature to the mathematical optimization the notion of invexity for differentiable functions, introduced by Hanson (1981) in [4]. He showed that a function is invex if and only if all stationary point is global minimizer.

Posteriorly Phuong, Sach and Yenin [6] consider the class formed by all locally Lipschitz real-valued functions and they introduce the following notion of invexity.

Definition 6. Let $\phi: \Omega \subseteq \mathbf{R}^{n} \rightarrow \mathbf{R}$ be a function defined on the open set $\Omega$ and $C \subset \Omega$ non-empty. We say that $\phi$ is invex on $C$ if for any $x, y \in C$ there exists a vector $\eta(y, x) \in T_{C}(x)$ such that

$$
\phi(y)-\phi(x) \geq \phi^{0}(x ; \eta(y, x)) .
$$

In [6] are obtained similar results to those obtained by Hanson. It is shown that a locally Lipschitz function $\phi: \Omega \subseteq \mathbf{R}^{n} \rightarrow \mathbf{R}$ is invex on $C$, if and only if every stationary point of $\phi$ in $C$ is a global minimizer.

This notion will be used to obtain the main results of this article.

\section{Results}

The next theorem establishes sufficient conditions to ensure that every feasible point is a properly efficient point.

Theorem 1. Let $X \neq \emptyset$. Assume that the objective functions $f_{j}, j \in I$ are locally Lipschitz in an open containing to $X$ and invex with respect to the same $\eta$, defined on $X$, with $\eta(x, y) \neq 0, \forall x \neq y$. Assume that there exist constants $k_{1}, k_{2}, 0<k_{1}<k_{2}$ such that for each $x \in X$

$$
k_{1} \leq\left\|\xi_{j}\right\| \leq k_{2}, \forall \xi_{j} \in \partial f_{j}(x), \quad j \in I
$$

and that there exists $\varepsilon>0$ such that $\forall x \in X$ and $\forall y \in T_{X}(x)$, with $\|y\|=1$, there exists $j \in I$ such that

$$
f_{j}^{0}(x ; y) \geq \varepsilon\left\|\xi_{j}\right\|,
$$

with $\xi_{j} \in \partial f_{j}(x)$. Then every point $x \in X$ is properly efficient solution of (P).

Proof: Initially we will prove that for every $i \in I$, there exist $d>0$ and $j \in I$ such that 


$$
f_{i}^{0}(x ; y)+d f_{j}^{0}(x, y) \geq 0, \forall x \in X, y \in \mathbf{B},
$$

where $\mathbf{B}:=\left\{y \in T_{X}(x),\|y\|=1\right\}$.

Indeed, let $x \in X$ be fixed then for each $y \in \mathbf{B}$ there exists $j \in I$ such that $f_{j}^{0}(x ; y) \geq \varepsilon\left\|\xi_{j}\right\|$, for some $\xi_{j} \in \partial f_{j}(x)$.

Let $S=\bigcup_{x \in X} S(x)$ be, where

$$
S(x)=\left\{-\frac{f_{i}^{0}(x ; y)}{f_{j}^{0}(x, y)}, i \in I(x, y)\right\}, y \in \mathbf{B}, j \in I,
$$

where $I(x, y)=\left\{i \in I: f_{i}^{0}(x ; y) \leq 0\right\}$.

Let us note that $S(x) \neq \emptyset$ for every $x \in X$ and consequently $S \neq \emptyset$. Moreover, for arbitrary values $z \in S(x)$ is fulfilled

$$
0 \leq z=-\frac{f_{i}^{0}(x ; y)}{f_{j}^{0}(x, y)} \leq-\frac{f_{i}^{0}(x ; y)}{\varepsilon\left\|\xi_{j}\right\|} \leq \frac{1}{\varepsilon}\left[-\frac{f_{i}^{0}(x ; y)}{k_{1}}\right] .
$$

As for each $\xi_{i} \in \partial f_{i}(x), y \in \mathbf{B},\left\langle\xi_{i}, y\right\rangle \leq f_{i}^{0}(x ; y)$ then for $i \in I(x, y)$

$$
0 \leq-f_{i}^{0}(x ; y) \leq-\left\langle\xi_{i}, y\right\rangle \leq\left|\left\langle\xi_{i}, y\right\rangle\right| \leq\left\|\xi_{i}\right\| \leq k_{2} .
$$

From (3.3) and (3.4), we get $0 \leq z \leq \frac{k_{2}}{k_{1} \varepsilon}, \forall z \in S$, this implies that $S$ is bounded. Therefore there exists $d=\sup S>0$ such that the condition (3.2) is fulfilled.

It will now proved that all point $x^{*} \in X$ is properly efficient solution of (P). Consider $d=\sup S>0, x^{*} \in X$ and $i \in I$ fixed. We want to prove that for each $x \in X, x \neq x^{*}$ there exists $j$ such that

$$
f_{i}(x)+d f_{j}(x) \geq f_{i}\left(x^{*}\right)+d f_{j}\left(x^{*}\right) .
$$

Take $x \in X, x \neq x^{*}$ and defined $y=\frac{\eta\left(x, x^{*}\right)}{\left\|\eta\left(x, x^{*}\right)\right\|} \in T_{X}\left(x^{*}\right)$. From inequality (3.2) and the fact $f_{j}^{0}\left(x^{*} ; \cdot\right)$ is positively homogeneous, it follows

$$
\frac{1}{\left\|\eta\left(x, x^{*}\right)\right\|}\left[f_{i}^{0}\left(x^{*} ; \eta\left(x, x^{*}\right)\right)+d f_{j}^{0}\left(x^{*} ; \eta\left(x, x^{*}\right)\right)\right] \geq 0,
$$

and thereby

$$
f_{i}^{0}\left(x^{*} ; \eta\left(x, x^{*}\right)\right)+d f_{j}^{0}\left(x^{*} ; \eta\left(x, x^{*}\right)\right) \geq 0 .
$$

As $f_{i}, f_{j}$ are invex functions with respect to $\eta$ on $X$ 


$$
\begin{array}{r}
f_{i}(x)-f_{i}\left(x^{*}\right) \geq f_{i}^{0}\left(x^{*} ; \eta\left(x, x^{*}\right)\right) \\
f_{j}(x)-f_{j}\left(x^{*}\right) \geq f_{j}^{0}\left(x^{*} ; \eta\left(x, x^{*}\right)\right)
\end{array}
$$

If we multiply by $d>0$ the second inequality and sum both inequalities obtained

$$
\left[f_{i}(x)+d f_{j}(x)\right]-\left[f_{i}\left(x^{*}\right)+d f_{j}\left(x^{*}\right)\right] \geq f_{i}^{0}\left(x^{*} ; \eta\left(x, x^{*}\right)\right)+d f_{j}^{0}\left(x^{*} ; \eta\left(x, x^{*}\right)\right) \geq 0 .
$$

This last inequality implies the condition (3.5). Thus $x^{*}$ is properly efficient solution $\mathbf{( P )}$.

The following result establishes under weaker hypothesis that all points feasible are efficient solutions of $(\mathbf{P})$.

Proposition 2. Let $X$ be a non-empty subset of $\mathbf{R}^{n}$. Assume that the objective functions $f_{j}, j \in I$ are locally Lipschitz in an open set containing to $X$, invex with respect to the same $\eta$ on $X$, with $\eta(x, y) \neq 0$, for all $x \neq y$ and that for each $x \in X$ and $y \in T_{X}(x) \backslash\{0\}$ there exists $j \in I$ such that $f_{j}^{0}(x ; y)>0$. Then every point $x \in X$ is efficient solution of $(\mathbf{P})$.

Proof: Let $x^{*} \in X$. Given $x \in X, x \neq x^{*}$ and $y=\eta\left(x, x^{*}\right) \in$ $T_{X}\left(x^{*}\right) \backslash\{0\}$ there exists $j \in I$ such that $f_{j}\left(x ; \eta\left(x, x^{*}\right)\right)>0$ and as all $f_{j}, j \in I$, are invex with respect to $\eta$, then

$$
f_{j}(x)-f_{j}\left(x^{*}\right) \geq f_{j}^{0}\left(x^{*} ; \eta\left(x, x^{*}\right)\right)>0 .
$$

Therefore there is no $x \in X$ such that $f\left(x^{*}\right) \leq f(x)$ and according to Definition 1, it follows that $x^{*}$ is efficient solution of $(\mathbf{P})$.

\section{Conclusions}

We have established sufficient conditions to ensure that all points of domain $X$ are properly efficient solutions (efficient solutions), for multi-objective optimization problems involving invex, locally Lipschitz functions. In future works, multi-objective optimal control problems will be studied. 


\section{References}

[1] V. Chankong, Y. Haimes: Multiobjective decision making: theory and methodology. North-Holland, New York, (1983).

[2] F. H. Clarke,Optimization and nonsmooth analysis, SIAM, Philadelphia, (1990).

[3] A. M. Geoffrion, Proper efficiency and the theory of vector maximization, J. Math. Anal. Appl., 22, pp. 618-630, (1968).

[4] M. A. Hanson, On sufficiency of the Kuhn-Tucker conditions. J. Math. Anal. Appl. 80, pp. 545-550, (1981).

[5] V. Pareto, Cours dÉconomie Politique, Rouge, (1886).

[6] T. D. Phuong, P. H. Sach, N. D. Yen, Strict lower semicontinuity of the level sets and invexity of a locally Lipschitz function, J. Optim. Th. Appl. 87, pp. 579-594, (1995).

[7] A. Siposová, A note on global Pareto optimality in multicriteria optimization problems, Nonlinear Analysis 69, pp. 1321-1324, (2008).

\section{Batista dos Santos}

Dpto. de Matemática,

Centro Politécnico,

Universidade Federal do Paraná, CEP 81531-980, CP 19081, Curitiba-PR,

Brasil

e-mail : lucelina@ufpr.br

\section{A. Rojas-Medar}

Instituto de Alta Investigación, Universidad de Tarapacá, Casilla 7D, Arica, Chile

e-mail : marko.medar@gmail.com

and 
438 L. Batista dos Santos, M.A. Rojas-Medar and V. Vivanco-Orellana

\author{
V. Vivanco-Orellana \\ Dpto. de Matemática y Física Aplicadas, \\ Facultad de Ingeniería, \\ Universidad Católica de la Santísima Concepción, \\ Concepción, \\ Chile \\ e-mail : vvivanco@.ucsc.cl
}

